\title{
Evaluation of a Novel Laboratory Candiduria Screening Protocol in the Intensive Care Unit
}

This article was published in the following Dove Press journal:

Infection and Drug Resistance

\author{
Zhengxin $\mathrm{He} \mathbb{D}^{1}{ }^{1} *$ \\ Chang $\mathrm{Su}^{2 *}$ \\ Yuwang $\mathrm{Bi}^{3}$ \\ Yan Cheng $\mathbb{D}^{\prime}$ \\ Daxin Lei ${ }^{1}$ \\ Fukun Wang'
}

'Laboratory Medicine, The 980th

Hospital of PLA Joint Logistical Support

Force (Bethune International Peace

Hospital), Shijiazhuang, Hebei, 050082 ,

People's Republic of China;

${ }^{2}$ Cardiothoracic Surgery, The 980th Hospital of PLA Joint Logistical Support Force (Bethune International Peace Hospital), Shijiazhuang, Hebei, 050082,

People's Republic of China; ${ }^{3}$ Information Center, The 980th Hospital of PLA Joint Logistical Support Force (Bethune International Peace Hospital),

Shijiazhuang, Hebei, 050082, People's

Republic of China

*These authors contributed equally to this work
Correspondence: Chang Su; Fukun Wang Tel +863 II87978349; +863 I I 87978537 Email suchang987@163.com; wangfk8@sina.com
Background: Since urine cultures are only guaranteed for patients with obvious urinary symptoms in most cases, most of candiduria episodes are ignored in clinic.

Objective: This study aimed to design a screening protocol to improve diagnostic efficiency of candiduria, and provide information of Candida species and drug susceptibility.

Methods: All patients, who were admitted to the intensive care unit (ICU) of our hospital during December 1, 2018 and October 1, 2019, were enrolled in this study. Urinalysis was performed every three days for each subject from the first day of ICU admission. Urine specimens were sampled for fungal culture with either condition: (1) yeast-like cell counting (YLCC) $\geq 200$; (2) positive YLCCs were observed in two consecutive tests, and at least one YLCC $\geq 100$.

Results: The screening protocol dramatically improved the candiduria diagnostic rate of ICU patients from $2.28 \%$ to $17.27 \%$. However, compared to the historical control, the screening protocol has no time-saving advantage in candiduria diagnosing. Higher percentage of $C$. albicans in screening protocol-identified candiduria patients was observed, although there was no statistical difference. Our results indicated that female gender, pneumonia, diabetes and infarction/hemorrhage patients were more prone to develop candiduria. Non-candiduria patients showed a better tendency for survival and shorter ICU stay length. Multisite colonization was common in the surveyed candiduria patients, who were up to $70.83 \%$ showed Candida positive cultures in sputum.

Conclusion: The screening protocol established in the study was a convenient and practical tool for early warning and feasible management of candiduria and IC.

Keywords: candiduria, intensive care unit, screening protocol, urinalysis, yeast-like cell counting

\section{Introduction}

Candiduria is defined as the presence of Candida species in the urine. Usually, candiduria is typically asymptomatic and rare in healthy patients, but it is more common in hospitalized patients and especially those in intensive care units (ICU). ${ }^{1,2}$ As our previous study indicated, the candiduria incidence rate is as high as $1.61 \%$ in hospitalized patients and $22.89 \%$ in ICU patients. ${ }^{3}$ Diabetes, urinary catheter, female gender, antibiotics, major abdominal surgery and ICU admission are considered as the important risk factors for candiduria., ${ }^{4,5}$ Most researchers believe that candiduria is an ominous characteristic sign of serious underlying conditions. The mortality rate in candiduria ICU patients is about 3 times higher than those without candiduria. $^{3,6}$ Candiduria is not only a marker for seriously ill patients that need particular attentions, but also an independent risk factor for invasive candidiasis 
(IC). ${ }^{7}$ For example, the Infectious Diseases Association of America (IDSA) guidelines (2016 version) emphasized the importance of treatment for candiduria patients under highrisk candidiasis conditions. A molecular correlation between Candida strains isolated from candidemia and concomitant candiduria indicated that hematogenous dissemination possibly existed in ICU candiduria patients. ${ }^{8}$

For clinical laboratories, urine culture is still the gold standard method for the candiduria diagnosis. Overall, urine culture is not sensitive enough and generally consumes about 2-5 days to achieve conclusive results. ${ }^{9}$ Lamentably, the slow turnaround time usually leads to the delayed therapeutic interventions and poor outcomes of candiduria patients. Furthermore, urine culture is generally only guaranteed when patient show symptoms of urinary tract infection (UTI) in, which means most of candiduria cases might be ignored in clinical practices.

Yeast-like cell counting (YLCC) is a parameter of automatic urinalysis system, which is shown as a reliable application for fungal detection in urine in several studies. ${ }^{10-12}$ Our previous work showed that YLCC is suitable for identifying urines with relatively high Candida CFUs. ${ }^{13}$ YLCCpositive urinalysis could imply candiduria prior to urine culture, especially the persistent positive YLCC cases. However, YLCC of urinalysis alone cannot provide sufficient information for candiduria managing, such as the
Candida species identification and yeast susceptibility test. In the present study, we therefore designed a convenient candiduria screening protocol for the potential candiduria patients. The advantages of YLCC and urine culture were combined together in the protocol to significantly enhance the efficiency of the candiduria identifying in ICU.

\section{Materials and Methods Study Design}

This was a single-center perspective cohort study aiming to validate the efficiency of a candiduria screening protocol in ICU. All patients, who were admitted to the ICU of our hospital during December 1, 2018 and October 1, 2019, were enrolled in this study. The schematic of the candiduria screening protocol is shown in Figure 1. Briefly, urinalysis was performed every three days for each subject from the first day of ICU admission. Urine samples were applied to fungal culture at either condition: (1) YLCC $\geq 200$; (2) positive YLCCs were observed in two consecutive tests, and at least one $\geq 100$. Notably, since the objective of this study was not intended to impact ICU patient care or management, physicians were informed about the fungal culture results only if the necessities were confirmed. Patient characteristics and management data were abstracted from medical records after ICU discharge or death.

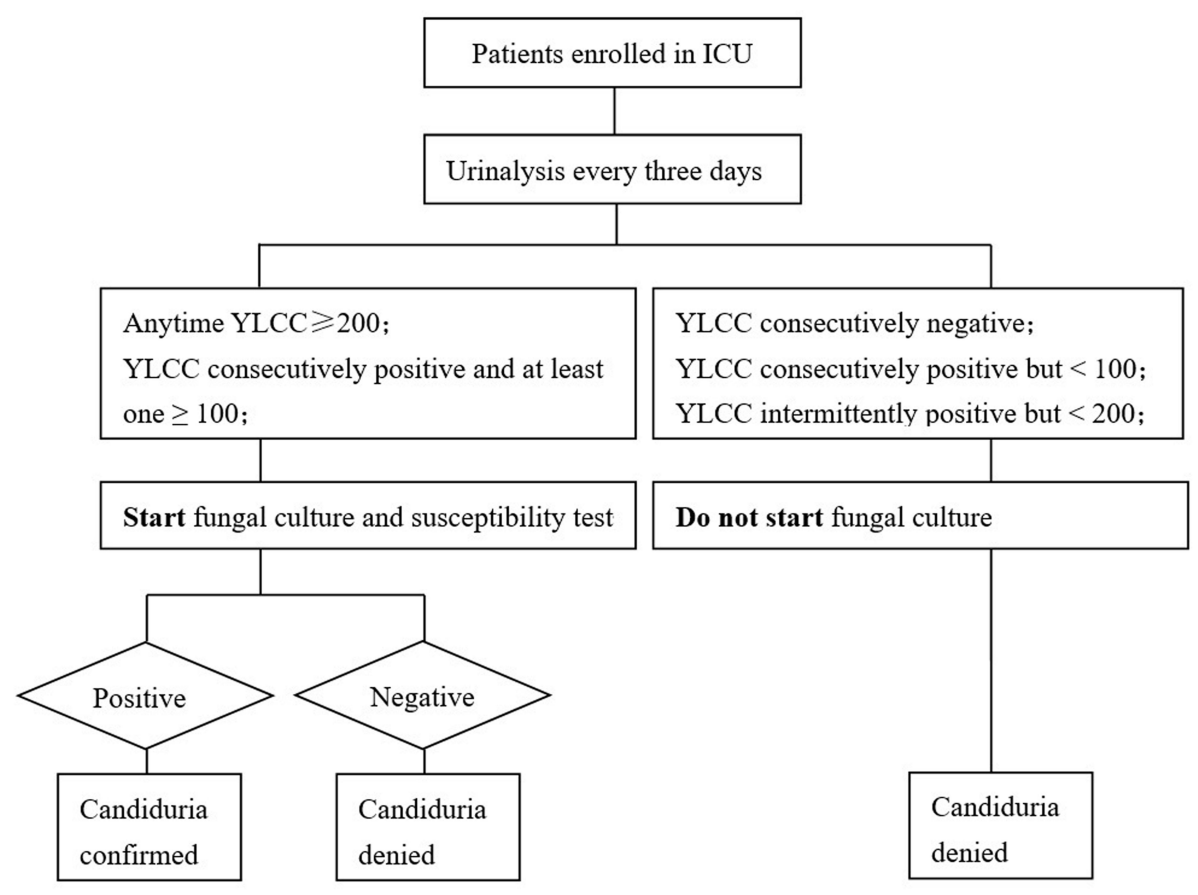

Figure I The schematic of the candiduria screening protocol designed in this study. 
To assess the diagnostic efficiency and Candida species distribution, the medical records of 1007 ICU patients admitted in our hospital between May 1st 2016 and November 30th 2018 were retrospectively reviewed and served as a historical control.

\section{Clinical Urinalysis}

Urine samples were firstly analyzed using the fully automatic UF-1000i system. Date, time and the urine collection method were registered by the attending nurse. All the urine samples were collected from the indwelling urine bags of the ICU patients. Hand washing was mandatory before the specimen collection and the external orifice of catheter was sterilized by $10 \%$ iodophor using a cotton swab for two times. Then, about $10 \mathrm{~mL}$ of urine was collected with a sterile syringe and stored in a disposable clean bottle with a screw top lid. All samples were analysed within 3 hours after the sample collection. The performance of urinalysis was conducted following the manufacturer's instruction in an ISO9000 certified laboratory.

\section{Isolate Identification and Antifungal Susceptibility Test}

YLCC positive urine specimens were applied to microbial cultivation to identify Candida species. Briefly, $10 \mu \mathrm{L}$ of urine was plated onto Candida chromogenic agar (CHROMagar ${ }^{\mathrm{TM}}$, Paris, France) using a half-plate quantitative urine streaking method. Plates were incubated at $35^{\circ} \mathrm{C}$ for $48 \mathrm{~h}$. The Candida species were screened based on the growth color and colony shape. Further fungal characterization and in vitro susceptibility tests were performed by a MA120 automated ID \& Ast system (Meihua Med Tech, Zhuhai, China), compliant with the manufacturer instructions.

The MA120 system is an automatic identification system which works based on the conventional biochemical reaction. Multi-charge coupled device (CCD) detection technology, multiple algorithms and advanced expert system are applied to ensure the validity of test results. Susceptibility test was carried out according to the Clinical \& Laboratory Standards Institute (CLSI) (2016) drug sensitivity standard. The system has passed the registration and certification of Guangdong Drug Administration of the China (No.: $20,152,220,508)$. The effectiveness of microbial identification and susceptibility test was confirmed by comparing with 16S rDNA sequencing and the VITEK 2 system. ${ }^{14}$

\section{Statistical Analysis}

Statistical analysis was performed with GraphPad Prism 7.00. For continuous variables, the Student's $t$-tests and Mann-Whitney U-tests were applied at the significant level $\mathrm{P}<0.05$. Categorical variables are described by percentage, which was studied using the Chi-square test or Fisher's exact test at the significance $\mathrm{P}<0.05$.

\section{Results}

\section{YLCC Combined with Yeast Culture} Improved Candiduria Screening Efficiency In this study, 615 urinalysis tests were totally carried out for the 139 patients, in which 24 candiduria episodes were confirmed by the screening protocol. All candiduria samples confirmed by the YLCC- criteria were culture positive, and the identified incidence rate for candiduria is $17.27 \%$ (24/139). For the historical control group, 23 candiduria episodes were confirmed by the physician prescribing urine culture empirically. Thus, the resultant rate of candiduria incidence identified by the conventional procedure was $2.28 \%$, which is significantly $(\mathrm{P}<0.0001)$ lower than that indicated by our protocol. Moreover, there was no significant difference in the interval time from ICU admission to confirmed candiduria for screening protocol group (median 15; inter-quartile range, 10-21) and the historical control group (median 10; inter-quartile range, 5-21) (Figure 2). Therefore, the screening protocol did not contribute to decrease the time-consuming for the candiduria diagnosis.

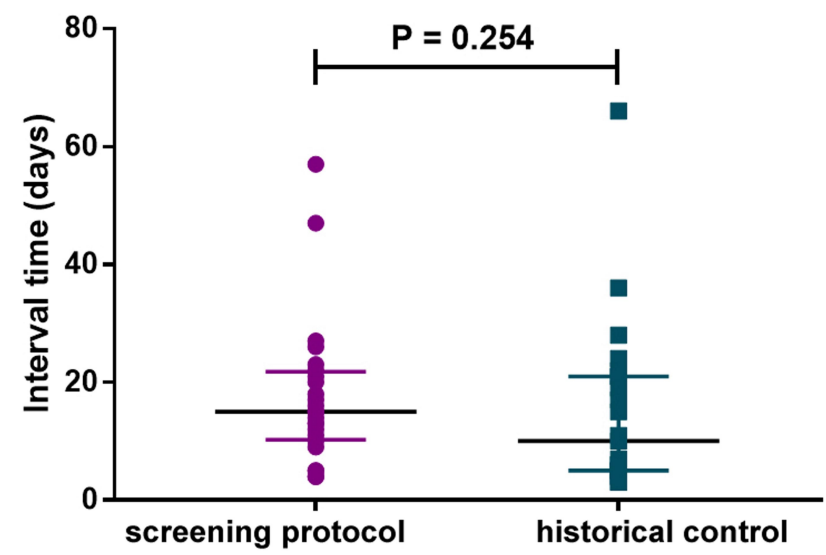

Figure 2 The interval times from ICU admission to confirmed candiduria by screening protocol and traditional pathway (historical control) are similar ( $\mathrm{P}$ $=0.254)$. 


\section{Clinical Characteristics of Candiduria and Non-Candiduria Patients}

Patients usually suffered from complicated diseases at the same time when they are admitted to ICU. In order to homogenize the cohort clinical characteristics, the enrolled patients were assessed by malignant tumor, diabetes, severe pneumonia, systematic infection, cardiac dysfunction, liver dysfunction, renal dysfunction, cerebral infarction/hemorrhage and surgical morbidities. Sequential organ failure assessment (SOFA) score was also recorded when a patient was admitted into the ICU ward. The patient characteristics and outcomes of the enrolled patients are listed in Table 1. Candiduria incidence rate was significant higher $(\mathrm{P}=$ 0.0070) in female gender than that in male. ICU patients with pneumonia, diabetes and infarction/ hemorrhage were more prone to develop candiduria. Compared to candiduria patients, non-candiduria patients showed a better tendency for survival and shorter ICU stay length.

Table I The Characteristics of the Patients Enrolled in This Study $(n=139)$

\begin{tabular}{|c|c|c|c|}
\hline \multirow[t]{2}{*}{ Characteristics } & \multicolumn{2}{|c|}{$\begin{array}{l}\text { Intensive Care Unit } \\
\text { Patients, N (\%) }\end{array}$} & \multirow[t]{2}{*}{$P$ value } \\
\hline & $\begin{array}{l}\text { Candiduria } \\
(n=24)\end{array}$ & $\begin{array}{l}\text { Non- } \\
\text { Candiduria } \\
(n=I \mid 5)\end{array}$ & \\
\hline Age, years & $69.58 \pm 18.99$ & $69.58 \pm 20.64$ & 0.4385 \\
\hline Female gender & $14(58.33)$ & $34(29.57)$ & $0.0070 *$ \\
\hline \multicolumn{4}{|l|}{ Basic conditions } \\
\hline SOFA score ${ }^{a}$ & $5.83 \pm 3.10$ & $6.69 \pm 3.17$ & 0.3955 \\
\hline Malignant tumor & $6(25.00)$ & $22(19.13)$ & 0.5143 \\
\hline Diabetes & $10(41.67)$ & $24(20.87)$ & $0.0311 *$ \\
\hline Pneumonia & $20(83.33)$ & $32(27.83)$ & $<0.0001 *$ \\
\hline Systematic infection & $4(16.67)$ & $22(19.13)$ & $>0.9999$ \\
\hline Respiratory failure & II (45.83) & $38(33.04)$ & 0.2329 \\
\hline Cardiac dysfunction & $13(54.17)$ & 39 (33.9I) & 0.0622 \\
\hline Liver dysfunction & $3(12.50)$ & $24(20.87)$ & 0.4116 \\
\hline Renal dysfunction & $2(8.33)$ & $32(27.83)$ & 0.0648 \\
\hline Infarction/hemorrhage & $10(41.67)$ & $12(10.43)$ & $0.0004^{*}$ \\
\hline Surgical morbidities & $4(16.67)$ & $40(34.78)$ & 0.0956 \\
\hline \multicolumn{4}{|l|}{ Outcomes } \\
\hline Dead in hospital & $12(50.00)$ & $19(16.52)$ & $<0.0001 *$ \\
\hline $\begin{array}{l}\text { ICU stay length } \\
\text { (median, range) }\end{array}$ & $21(17-98)$ & $10(1-108)$ & $0.0002 *$ \\
\hline
\end{tabular}

Note: $* p<0.05$.

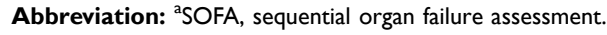

\section{Distribution and Drug Sensitivity of}

\section{Candida Species}

In this study, 24 candiduria cases were, respectively, identified the different Candida species including 12 strains (50\%) of C. albicans, 4 strains (16.67\%) of C. tropicalis, 4 strains (16.67\%) of C. glabrata and 4 strains $(16.67 \%)$ of C. parapsilosis were identified. For the 23 historical control candiduria cases which were confirmed by conventional clinical procedure, the proportion of C. albicans, C. tropicalis, C. glabrata and C. parapsilosis accounted for $30.43 \%$, $34.78 \%, 21.74 \%$ and $13.04 \%$, respectively (Figure 3). Specially, the percentage of $C$. albicans associated candiduria was higher (50\% VS 30.43\%) in the screening protocol group although there was no statistical difference between this study and the historical control group.

Antifungal susceptibility testing was carried out on the 24 Candida isolates that were identified in the study. As indicated in Table 2, all yeast strains were mostly susceptible to fluorocytosine (24/24), voriconazole (23/24) and amphotericin B (23/24). But the rates of fungal resistance to fluconazole, itraconazole and caspofungin were relatively higher, especially in C. glabrata. Interestingly, we identified a multidrugresistant $C$. tropicalis isolate which was resistant to fluconazole, voriconazole, itraconazole, amphotericin B and caspofungin.

\section{Candiduria and Multisite Candida Colonization/Infection}

Multisite Candida colonization/infection was found to be highly prevalent in the ICU candiduria patients. We reviewed the records of sputum culture collected from the 24 candiduria patients identified by the screening protocol. Seventeen $(70.83 \%)$ of the candiduria patients showed positive sputum culture for Candida (Table 3). In 10 cases, the positive Candida cultures in sputum were earlier reported than those

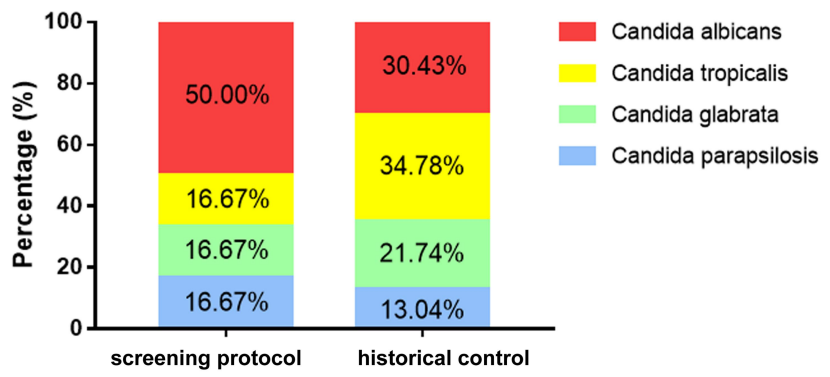

Figure 3 Candida species distribution in groups of patients identified by screening protocol and historical control. Percentage of $C$. albicans was higher in the screening protocol group than the historical control group. However, the difference was not significant. 
Table 2 Antifungal Susceptibility Testing Results for Common Candida Species Causing Candiduria Reviewed in This Study ( $=24$ )

\begin{tabular}{|c|c|c|c|c|c|}
\hline \multirow[t]{2}{*}{ Organism (n) } & \multirow[t]{2}{*}{ Antifungal Agent } & \multirow[t]{2}{*}{ MIC Range (mg/L) } & \multicolumn{3}{|c|}{ No. of Strains } \\
\hline & & & Susceptible & $\% S-D D / I^{\mathbf{a}}$ & Resistant \\
\hline \multirow[t]{6}{*}{ C. albicans (12) } & Fluorocytosine & $\leq 2$ & 12 & 0 & 0 \\
\hline & Fluconazole & $\leq 2$ & 12 & 0 & 0 \\
\hline & Voriconazole & $\leq 0.12$ & 12 & 0 & 0 \\
\hline & Itraconazole & $\leq 0.06$ to $>2$ & 10 & 1 & I \\
\hline & Amphotericin B & $\leq 0.25$ to $\mathrm{I}$ & 12 & 0 & 0 \\
\hline & Caspofungin & $\leq 0.12$ to $\mathrm{I}$ & 3 & 8 & 1 \\
\hline \multirow[t]{6}{*}{ C. tropicalis (4) } & Fluorocytosine & $\leq 2$ & 4 & 0 & 0 \\
\hline & Fluconazole & $\leq 2$ to $>64$ & 3 & 0 & 1 \\
\hline & Voriconazole & $\leq 0.12$ to 2 & 3 & 0 & 1 \\
\hline & Itraconazole & $=0.25$ to $>2$ & 0 & 2 & 2 \\
\hline & Amphotericin B & $\leq 0.25$ to 2 & 3 & 0 & 1 \\
\hline & Caspofungin & $\leq 0.12$ to $I$ & 2 & 1 & I \\
\hline \multirow[t]{6}{*}{ C. glabrata (4) } & Fluorocytosine & $\leq 2$ & 4 & 0 & 0 \\
\hline & Fluconazole & $\leq 2$ to 64 & 1 & 2 & 1 \\
\hline & Voriconazole & $\leq 0.12$ to 0.25 & 4 & 0 & 0 \\
\hline & Itraconazole & 0.12 to 1 & I & I & 2 \\
\hline & Amphotericin B & 0.5 to 1 & 4 & 0 & 0 \\
\hline & Caspofungin & 0.25 to 0.5 & 0 & 2 & 2 \\
\hline \multirow[t]{6}{*}{ C. parapsilosis (4) } & Fluorocytosine & $\leq 2$ & 4 & 0 & 0 \\
\hline & Fluconazole & $\leq 2$ to 8 & 3 & 0 & 1 \\
\hline & Voriconazole & $\leq 0.12$ & 4 & 0 & 0 \\
\hline & Itraconazole & 0.12 to 0.25 & 2 & 2 & 0 \\
\hline & Amphotericin B & 0.5 & 4 & 0 & 0 \\
\hline & Caspofungin & $\leq 0.12$ to $I$ & 4 & 0 & 0 \\
\hline
\end{tabular}

Abbreviations: ${ }^{\mathrm{S} S-D D}$, susceptible dose dependent; I, intermediate.

in urine. Same Candida species cultures were obtained both in sputum and urine of 7 patients. Antifungal therapies were initiated for 19 patients, in which 14 patients were confirmed candiduria prior to the therapies. Moreover, 4 of the 139 enrolled patients were diagnosed with invasive candidiasis (IC) (3 with positive blood cultures and 1 with positive peritoneal fluid culture). Remarkably, all of these IC patients had candiduria before diagnosed, and 3 of them had been identified the same Candida species in their sputum, urine and sterile body fluid.

\section{Discussion}

YLCC is a urinalysis parameter which is easy to conduct and obtain in clinical laboratories. Previous studies have shown that this parameter has the potential to screen candiduria patients efficiently. ${ }^{13,15,16}$ Particularly, this prospective study was primarily designed to answer the combination of YLCC and urine microbial culture can achieve the rapid and effective identification of candiduria and Candida species. The screening protocol improved the candiduria 
Table 3 Characteristics of Multisite Colonization for the 24 Candiduria Patients Identified in This Study

\begin{tabular}{|l|l|}
\hline Characteristics & $\begin{array}{l}\text { No. of } \\
\text { Patients (\%) }\end{array}$ \\
\hline Multisite colonization & $17(70.83)$ \\
\hline Same Candida species in urine and sputum & $7(29.17)$ \\
\hline Sputum culture positive before urine & $10(45.83)$ \\
\hline Urine culture positive before antifungal therapy & $14(58.33)$ \\
\hline Evidence of invasive candidiasis $^{\text {b }}$ & $4(16.67)$ \\
\hline
\end{tabular}

Notes: ${ }^{a}$ Calculating percentages, the denominator was the 24 candiduria patients; ${ }^{b} 3$ with positive blood cultures and I with positive peritoneal fluid culture.

diagnostic rate ranged from $2.28 \%$ to $17.27 \%$ in ICU, which is consistently similar to our previous retrospective analysis. ${ }^{3}$ In the traditional course of the clinical diagnosis and treatment, physicians usually tend to focus on candiduria patients with obvious UTI symptoms. Most candiduria patients, especially those asymptomatic cases, are ignored by the management path. Indeed, it is reasonable to suspect that a part of candiduria clinical investigations based on traditional pathway may be affected by such ignorance. ${ }^{17}$ Compared with the historical control cases, the screening protocol here has no time-saving advantage in candiduria diagnosing. The reason may be that the candiduria cases diagnosed by traditional pathway often show UTI symptoms in the early stage of ICU admission, while most cases diagnosed by screening protocol are secondary and asymptomatic.

According to the screening protocol with the higher candiduria diagnosing efficiency and a larger number of candiduria population in ICU, we tried to describe the characteristics and clinical outcomes of the candiduria patients. Female gender and diabetes were associated with candiduria, which was consistent with many previous studies. ${ }^{18,19}$ We have found that pneumonia patients were prone to develop candiduria in another retrospective study, ${ }^{20}$ which was once again confirmed in this observational study. We may speculate the fact that the ICU pneumonia patients often experience a long period of intensive use of antibiotics, which has been recognized as an important risk factor for candiduria. ${ }^{21,22}$ While for infarction/hemorrhage patients, the longer ICU stay length may be the main cause of candiduria. ${ }^{1}$ Numerous previous reports have indicated that candiduria was a marker for poor clinical prognosis. ${ }^{3,23,24}$ It is therefore not suspiring that we observed the higher mortality (more than 3 times) and the longer ICU stay length in candiduria patients.

Our findings showed the higher proportion of C. albicans in the screening protocol-identified candiduria patients, without the statistical difference. Differed from the candiduria patients confirmed by traditional pathway, most of candiduria patients diagnosed in this study were asymptomatic. Previous studies have produced mixed results on which Candida species is more likely to cause UTIs. C. albicans, ${ }^{25}$ C. tropicalis $^{26,27}$ and C. glabrata $^{19}$ have been, respectively, considered to be more associated with urinary infection in the different reports. Thus, the differentiation of Candida species distribution of urinary Candida colonization and infection is an issue worthy of discussion in future clinical research.

Another important finding of this study was the fact that multisite colonization was common in candiduria patients. Up to $70.83 \%$ of candiduria patients had Candida positive cultures in sputum. Candida colonization is considered to be associated with IC, and multisite colonization is an important risk factor in clinic. ${ }^{20,28}$ Several risk assessment tools including colonization index $(\mathrm{CI}),{ }^{29}$ corrected colonization index $(\mathrm{CCI})^{30}$ and Candida score $(\mathrm{CS})^{31}$ are used to evaluate the possibility of IC in critical patients. Among these tools, multisite Candida colonization is an important clinical reference index. Though only two sites of ICU patients were investigated in this study, a considerable number of multisite colonization candiduria cases were found, especially 4 cases of evidence-based IC. We speculated that the incidence of multisite colonization and IC may be higher than is generally thought.

In conclusion, our protocol essentially moved the candiduria screening standard from urinary symptoms to urinalysis data, which greatly enhanced the efficiency of candiduria diagnosing. We believe this convenient and practical protocol play important implications for early warning and management of candiduria and IC.

\section{Ethical Approval}

The authors are accountable for all aspects of the work in ensuring that questions related to the accuracy or integrity of any part of the work are appropriately investigated and resolved. The trial was conducted in accordance with the Declaration of Helsinki (as revised in 2013). The study was approved by institutional ethics board of 980th Hospital of PLA Joint Logistical Support Force (Bethune International 
Peace Hospital) (NO. 2018-KY-32) and informed consent was taken from all individual participants.

\section{Acknowledgments}

This work was supported by grants from the Key R\&D Program Projects of Hebei Province (No. 19277771D), the Science Foundation of Bethune International Peace Hospital of PLA (No. 20150014) and the Science and Technology Research and Development Plan of Shijiazhuang (No. 201200623).

\section{Disclosure}

The authors declare that they have no conflicts of interest.

\section{References}

1. Alfouzan WA, Dhar R. Candiduria: evidence-based approach to management, are we there yet? J Mycol Med. 2017;27(3):293-302. doi:10.1016/j.mycmed.2017.04.005

2. Gajdács M, Dóczi I, Ábrók M, Lázár A, Burián K. Epidemiology of candiduria and Candida urinary tract infections in inpatients and outpatients: results from a 10-year retrospective survey. Cent European J Urol. 2019;72(2):209-214. doi:10.5173/ceju.2019.1909

3. He Z, Liu Y, Wang T, Cheng Y, Chen J, Wang F. Candiduria in hospitalized patients: an investigation with the Sysmex UF-1000i urine analyzer. PeerJ. 2019;7:e6935. doi:10.7717/peerj.6935

4. Jain M, Dogra V, Mishra B, Thakur A, Loomba PS, Bhargava A. Candiduria in catheterized intensive care unit patients: emerging microbiological trends. Indian J Pathol Microbiol. 2011;54 (3):552-555. doi:10.4103/0377-4929.85091

5. Kauffman CA, Vazquez JA, Sobel JD, et al. Prospective multicenter surveillance study of funguria in hospitalized patients. The National Institute for Allergy and Infectious Diseases (NIAID) mycoses study group. Clin Infect Dis. 2000;30(1):14-18. doi:10.1086/313583

6. Bougnoux ME, Kac G, Aegerter P, CandiRea Study Group. Candidemia and candiduria in critically ill patients admitted to intensive care units in France: incidence, molecular diversity, management and outcome. Intensive Care Med. 2008;34(2):292-299. doi:10.1007/ s00134-007-0865-y

7. Wang K, Hsueh K, Kronen R, et al. Creation and assessment of a clinical predictive model for candidaemia in patients with candiduria. Mycoses. 2019;62(7):554-561. doi:10.1111/myc.12917

8. Drogari-Apiranthitou M, Anyfantis I, Galani I, Kanioura L, Daikos GL, Petrikkos G. Association between candiduria and candidemia: a clinical and molecular analysis of cases. Mycopathologia. 2017;182(11-12):1045-1052. doi:10.1007/s11046-017-0180-2

9. Pitarch A, Nombela C, Gil C. Diagnosis of invasive candidiasis: from gold standard methods to promising leading-edge technologies. Curr Top Med Chem. 2018;18(16):1375-1392. doi:10.2174/156802 6618666181025093146

10. Gutiérrez-Fernández J, Riazzo C, Sanbonmatsu S, et al. Sysmex UF-1000i performance for screening yeasts in urine. APMIS. 2014;122(4):324-328. doi:10.1111/apm.12148

11. Herráez O, Asencio MA, Carranza R, et al. Sysmex UF-1000i flow cytometer to screen urinary tract infections: the URISCAM multicentre study. Lett Appl Microbiol. 2018;66(3):175-181. doi:10.1111/ lam. 12832

12. Le Z, Li F, Fei C, Ye A, Xie X, Zhang J. Performance of the Sysmex UF-1000i urine analyser in the rapid diagnosis of urinary tract infections in hospitalized patients. $J$ Infect Chemother. 2016;22 (6):377-382. doi:10.1016/j.jiac.2016.02.009
13. Zhengxin H, Haipu Z, Yan C, et al. Performance of yeast-like cell counting (YLCC) using the Sysmex UF-1000i for clinical candiduria screening. Eur J Clin Microbiol Infect Dis. 2019;38(5):891-894. doi:10.1007/s10096-019-03491-5

14. Zhang J, Zhang C, Jia T, et al. Effect evaluation of MA 120 microbial identification and drug sensitivity analysis system in clinical microbiology identification and drug sensitivity test (in Chinese). Infect Dis Info. 2016;29(5):267-271.

15. De Rosa R, Grosso S, Lorenzi G, Bruschetta G, Camporese A. Evaluation of the new Sysmex UF-5000 fluorescence flow cytometry analyser for ruling out bacterial urinary tract infection and for prediction of Gram negative bacteria in urine cultures. Clin Chim Acta. 2018;484:171-178. doi:10.1016/j.cca.2018.05.047

16. Ren $\mathrm{C}$, Jin $\mathrm{M}$, Wu J, Wang $\mathrm{X}$, Wang $\mathrm{Y}$, Cao $\mathrm{H}$. Improving the detection of urine sediment with a modified urinalysis review procedure. Clin Lab. 2019;65(4). doi:10.7754/Clin. Lab.2018.180830

17. Aubron C, Suzuki S, Glassford NJ, Garcia-Alvarez M, Howden BP, Bellomo R. The epidemiology of bacteriuria and candiduria in critically ill patients. Epidemiol Infect. 2015;143(3):653-662. doi:10. 1017/S0950268814000934

18. Safdar N, Slattery WR, Knasinski V, et al. Predictors and outcomes of candiduria in renal transplant recipients. Clin Infect Dis. 2005;40 (10):1413-1421. doi:10.1086/429620

19. Falahati M, Farahyar S, Akhlaghi L, et al. Characterization and identification of candiduria due to Candida species in diabetic patients. Curr Med Mycol. 2016;2(3):10-14. doi:10.18869/acadpub. cmm.2.3.10

20. He Z, Huo X, Lei D, Zhao H, Jia K, Wang F. Management of candiduria in hospitalized patients: a single-center study on the implementation of IDSA guidelines and factors affecting clinical decisions [published online ahead of print, 2020 Jul 30]. Eur J Clin Microbiol Infect Dis. 2020;40:59-65. doi:10.1007/s10096-020-03999-1.

21. Kumar S, Singhi S, Chakrabarti A, Bansal A, Jayashree M. Probiotic use and prevalence of candidemia and candiduria in a PICU. Pediatr Crit Care Med. 2013;14(9):e409-e415. doi:10.1097/ PCC.0b013e31829f5d 88

22. Padawer D, Pastukh N, Nitzan O, et al. Catheter-associated candiduria: risk factors, medical interventions, and antifungal susceptibility. Am J Infect Control. 2015;43(7):e19-e22. doi:10.10 16/j.ajic.2015.03.013

23. Nassoura Z, Ivatury RR, Simon RJ, Jabbour N, Stahl WM. Candiduria as an early marker of disseminated infection in critically ill surgical patients: the role of fluconazole therapy. $J$ Trauma. 1993;35(2):290-295. doi:10.1097/00005373-199308000-00020

24. Sobel JD, Fisher JF, Kauffman CA, Newman CA. Candida urinary tract infections-epidemiology. Clin Infect Dis. 2011;52(Suppl 6): S433-S436. doi:10.1093/cid/cir109

25. García-Agudo L, Rodríguez-Iglesias $\mathrm{M}$, Carranza-González R. Nosocomial candiduria in the elderly: microbiological diagnosis. Mycopathologia. 2018;183(3):591-596. doi:10.1007/s11046-0170232-7

26. Negri M, Silva S, Henriques M, Oliveira R. Insights into Candida tropicalis nosocomial infections and virulence factors. Eur $J$ Clin Microbiol Infect Dis. 2012;31(7):1399-1412. doi:10.1007/s10096011-1455-z

27. Santana MMP, Hoffmann-Santos HD, Dias LB, et al. Epidemiological profile of patients hospitalized with candiduria in the Central-Western region of Brazil. Rev Iberoam Micol. 2019;36 (4):175-180. doi:10.1016/j.riam.2019.04.006

28. Lau AF, Kabir M, Chen SC, et al. Candida colonization as a risk marker for invasive candidiasis in mixed medical-surgical intensive care units: development and evaluation of a simple, standard protocol. J Clin Microbiol. 2015;53(4):1324-1330. doi:10.1128/ JCM.03239-14 
29. Eggimann P, Pittet D. Candida colonization index and subsequent infection in critically ill surgical patients: 20 years later. Intensive Care Med. 2014;40(10):1429-1448. doi:10.1007/s00134-0143355-z

30. Caggiano G, Puntillo F, Coretti C, et al. Candida colonization index in patients admitted to an ICU. Int J Mol Sci. 2011;12 (10):7038-7047. doi:10.3390/ijms 12107038
31. León C, Ruiz-Santana S, Saavedra P, et al. A bedside scoring system ("Candida score") for early antifungal treatment in nonneutropenic critically ill patients with Candida colonization. Crit Care Med. 2006;34(3):730-737. doi:10.1097/01.CCM.0000202208.37364.7D

\section{Publish your work in this journal}

Infection and Drug Resistance is an international, peer-reviewed openaccess journal that focuses on the optimal treatment of infection (bacterial, fungal and viral) and the development and institution of preventive strategies to minimize the development and spread of resistance. The journal is specifically concerned with the epidemiology of antibiotic resistance and the mechanisms of resistance development and diffusion in both hospitals and the community. The manuscript management system is completely online and includes a very quick and fair peerreview system, which is all easy to use. Visit http://www.dovepress.com/ testimonials.php to read real quotes from published authors. 